\title{
Theory of the non-stationary arc column
}

\author{
R. L. PHILLIPS \\ Department of Aerospace Engineering, University of Michigan, Ann Arbor, Michigan, \\ U.S.A.
}

MS. received 16th May 1966, in revised form 31st August 1966

\begin{abstract}
The equations that describe the behaviour of a time-unsteady plasma column have been simplified by neglecting energy transfer by radiation and convection. Furthermore, the thermal diffusivity of the plasma was assumed to be constant and a simplified expression for the electrical conductivity was adopted. Thus the energy equation was cast into a universal form, requiring the specification of only two parameters to obtain solutions. Although some approximate closed form solutions were obtained it was necessary to solve the general a.c. arc problem on a digital computer. In view of the good agreement between the theoretically derived voltage waveforms and those found from a specially designed a.c. cascade it is concluded that the behaviour of the low current a.c. arc can be described by the solution of a complicated heat conduction problem. These solutions have the advantage over older theories that they can be directly related to measurable or calculable quantities that characterize a particular gas.
\end{abstract}

\section{Introduction}

Until recently studies of the electric arc have been in the domain of physicists and electrical engineers (Finkelnburg and Maecker 1956, Loh 1959). The advent of the ballistic missile, however, created the need for laboratory simulation of the high temperature re-entry environment and the aeronautical engineer quickly turned to the arc heater (or plasma jet) as a means of obtaining high enthalpy gas flows. In these devices the aim is to pass a stream of cold gas through and around a stabilized arc discharge thereby heating the flow to extreme temperatures. It is evident upon reviewing the literature (John and Bade 1961) that the development of the arc heater type of device has relied almost exclusively upon trial and error procedures. The complex interaction between the arc temperature field and the surrounding flow field, and the non-linear dependence of transport properties upon temperature have made analysis of the problem especially difficult.

The present work was initiated in an attempt to understand some of the fundamental processes that occur in an arc type of gas heater, specifically one that uses an alternating current power supply. The details of this device, as well as some analytical investigations of its behaviour have been reported (Phillips 1964a, b).

The theory of the arc as developed here entirely disregards any phenomena that are due to the presence of electrodes. As is customary in studies of this sort only the quasi-neutral arc column is considered, for in many cases this part of the discharge determines the characteristics of the whole arc. For the cylindrical d.c. arc column, it is a simple matter to formulate an energy balance known historically as the Elenbaas-Heller equation. Uhlenbusch (1962 Dissertation, Tech. Hochschule Aachen) presents a summary of the various approaches that have been used to solve this equation in the case of the wallstabilized d.c. arc. In general, the theory of the cylindrically symmetric arc is firmly established and compares well with experiment.

The analytical description of the energy transfer processes in dynamic arcs is complicated by the presence of the time variable. Most of the work on a.c. arcs has been done in connection with circuit breaker design, but nothing as comprehensive as that reported for d.c. arcs appears in the literature. In short, the theory of the a.c. arc is not well established. Two noteworthy attempts at a theoretical treatment of the dynamic, cylindrical, positive 
column were reported about 25 years ago by Cassie (1939) and Mayr (1943). Both of these investigators formulated arc models where the radial variation of properties was not included or was integrated away and not considered further. Cassie (1939), for example, assumes an arc column where the temperature is constant in space and time, having constant per-unit-volume resistivity, power loss and energy content. In order to obtain a variation in column conductance he invokes a variable arc radius. This leads to an ordinary differential equation with conductance as the dependent variable. Mayr (1943), on the other hand, defines an 'ersatz' are gas whose transport properties exhibit a Gaussian dependence upon the radial variable. An integration over the arc radius is then performed so that there remains only an ordinary differential equation for the arc conductance. If the assumption is made that the column energy loss per unit length is constant, it is possible to solve Mayr's equation for specified applied current or voltage variations. While the work of both Cassie and Mayr has had some limited usefulness for circuit breaker engineers, little insight into the detailed energy transfer processes that affect arc behaviour has been gained.

Other attempts (Bishop 1954, Noeske 1957, Edels and Ettinger 1962) to explain the nature of the dynamic arc are subject to the same objection put forth above; an integral approach is used to cast the governing partial differential equation into a simpler ordinary differential equation. The situation can be likened to a boundary layer theory based solely upon solutions of the Karman-Polhausen type with no knowledge of the exact profiles that exist.

From the theories of Cassie and Mayr there arises the notion of an arc time constant, a typical time required for thermal re-adjustment. Because of the oversimplified nature of the Cassie and Mayr models, however, and because the boundary conditions in either case are not clearly defined, it is not possible to relate this time constant to any of the known conditions under which an arc is operating. In the present work a theory is developed which contains a time constant that is easily related to known conditions. This is made possible, in part, by considering a wall-stabilized arc, i.e., one burning in a well-cooled tube. Frind's (1960) work in this area is noteworthy, but his time constants are applicable only to idealized, constant radius arcs.

In the present investigation a theory for the cylindrically symmetric, dynamic, wallstabilized arc has been formulated that considers the detailed structure of the column. A simplified electrical conductivity function was assumed so that the behaviour of a dynamic arc could be determined, independent of any type of gas. A set of non-linear partial differential equations resulted. In two special cases, for a high-frequency a.c. arc and for a d.c. arc that experiences a step function modulation in current, it was possible to obtain a closed form solution to the governing equations. In the general case, numerical solutions for the quasi-steady a.c. arc have been obtained with the aid of a digital computer.

In an earlier work (Phillips 1964 Dissertation, University of Michigan) it was shown that the theory in question applies not only to a stagnant arc column but also describes the portion of an arc far from the entrance region of tube-type arc heaters. This fact has made it possible for the author to compare the theoretically derived voltage waveforms with those obtained from a Maecker-type cascade employing axial gas flow. Good agreement in waveform shape and amplitude was obtained (Phillips 1964 Dissertation).

\section{The fundamental equations}

The equations needed to provide a description of the energy transfer processes within an electric arc are the well-established multi-component conservation equations. It is not intended to derive those equations here since they are exhaustively discussed in the literature (Hirschfelder, Curtiss and Bird 1954).

It is stipulated that no external magnetic fields will be applied to the region occupied by the arc column and, furthermore, the current carried by the arc will be small enough so that the self-magnetic field will be of no importance. In addition it is assumed that the arc gas is quasi-neutral. A result of the above stipulations is that little new knowledge is gained 
from Maxwell's equations and that the conservation equations combined with a suitable form of Ohm's law suffice to describe the are column properties completely.

If we neglect the effects of radiation, convection and chemical relaxation, it is possible to specialize the energy equation and Ohm's law to strict cylindrical symmetry. In the absence of charge separation and induced electric fields, the electric field in the arc has only an axial component and is independent of radius. It is therefore a function of time alone. The arc burns in a well-cooled tube of radius $R$ whose walls are maintained at a constant temperature, but which carry no current. In line with other investigators the diffusive contribution to energy transfer is combined with the 'frozen' thermal conductivity $k$ to give a total effective thermal conductivity $\bar{k}$. Thus the energy equation becomes

$$
\sigma(T)\{E(t)\}^{2}+\frac{1}{r} \frac{\partial}{\partial r}\left(r \bar{\kappa} \frac{\partial T}{\partial r}\right)=\rho(T) \frac{\partial h}{\partial t}
$$

where $0 \leqslant r \leqslant R, T(R, t)=T_{\mathrm{w}}$ and $t>0$. Here, $\sigma, T, \rho$ and $h$ are the electrical conductivity, temperature, density and the mixture enthalpy, respectively. In addition, Ohm"s law becomes

$$
E(t) \int_{0}^{R} 2 \pi r \sigma(T) d r=I(t)
$$

where $E$ and $I$ are the time dependent electric field and current. In this form the energy equation is simply a relation between the Joule heat produced in the arc column, the energy transport by conduction and the storage term $\rho \partial h / \partial t$. The electric field strength is understood to have the spatially constant axial value mentioned above. In general, an equation describing an electric circuit should accompany the above set, but it is presumed here that the arc is sustained by a current source, so that $I(t)$ occurring in Ohm's law is a specified function of time.

The temperature variation of the transport properties and the quadratic manner in which the electric field enters the problem introduce strong non-linearities, and unless one is willing to embark on a complete numerical programme for the solution of these equations, it is necessary to make some simplifications. We begin by introducing a transformation first used by Schmitz (1950). He defined the heat flux potential $S(T)$ by the relation

$$
S(T)=\int_{T_{\mathrm{ref}}}^{T} \bar{k}(a) d \alpha .
$$

Using $S$ instead of temperature as the dependent variable removes the thermal conductivity from the problem, but one must then express all other thermodynamic and transport

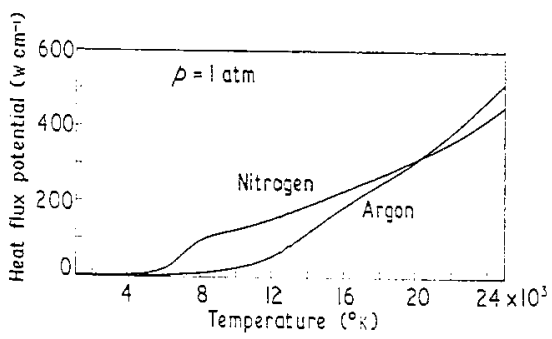

Figure 1. Temperature dependence of heat flux potential for nitrogen and argon.

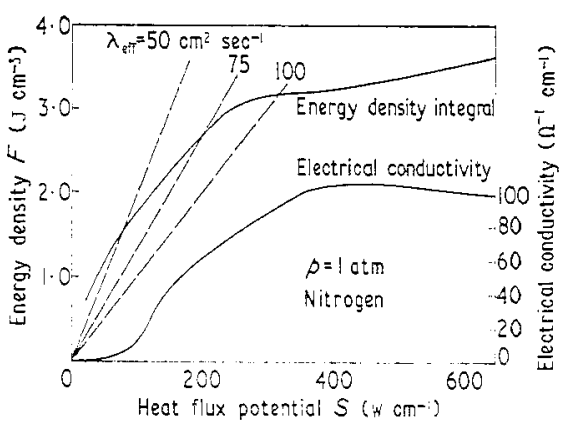

Figure 2. Electrical conductivity and energy density integral as a function of heat flux potential for nitrogen. Broken straight lines represent values of constant thermal diffusivity. 
properties in terms of $S$. The heat flux potential for nitrogen and argon is shown in figure 1. The pressure is one atmosphere and the data are due to John et al. (1963) and Marlotte (1963 private communication).

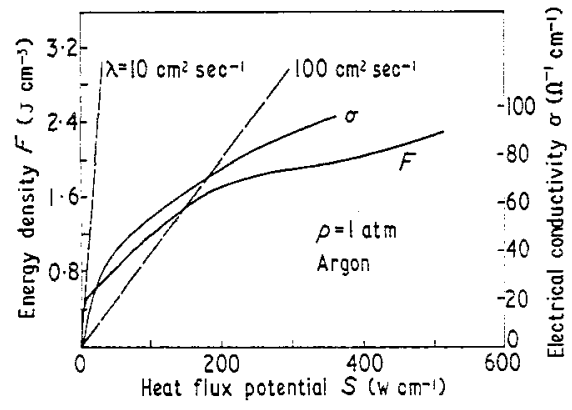

Figure 3. Electrical conductivity and energy density integral as a function of heat fiux potential for argon. Broken straight lines represent values of constant thermal diffusivity.

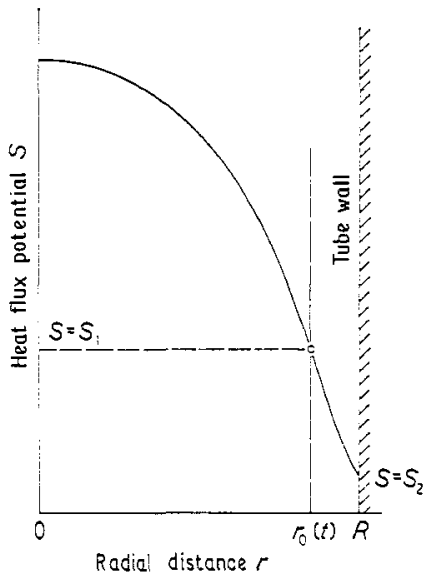

Figure 4. Coordinate system for the cylindrically symmetric arc.

There remains the problem of dealing with the electrical conductivity function. In figures 2 and 3 the electrical conductivity of nitrogen and argon is shown as a function of $S$ and it is seen in either case that $\sigma$ becomes non-zero quite abruptly and rises sharply to rather large values. In his studies of direct current arcs Maecker (1959) showed that approximating the $(\sigma, S)$ behaviour by two straight lines yields good results. Specifically we assume

$$
\begin{array}{ll}
\sigma=0, & S<S_{1} \\
\sigma=B\left(S-S_{1}\right), & S>S_{1}
\end{array}
$$

where $S_{1}$ and $B$ are appropriate constants.

While this model has some drawbacks it has the advantage of allowing the governing equations to be fully non-dimensionalized. Thus they can be solved in general with no reference to a particular gas. In addition, the sharp onset of electrical conductivity caused by this model introduces the notion of a conducting zone or arc radius, an extremely useful fiction. This radius is the position where the local heat flux potential is equal to $S_{1}$, the cut-off value, and for the a.c. arc it is a function of time. This latter fact introduces an interesting mathematical complication which will be discussed later.

Finally, in order that the proposed analysis not be tied to any specific gas, it is convenient to introduce a new integral variable $F$, defined by the relation

$$
F(T)=\int_{T_{\text {Ief }}}^{r} \rho(\alpha) C_{\mathrm{p}}(\alpha) d \alpha .
$$

This function can be easily computed (Drellishak et al. 1964) and one can prepare an $(F, S)$ plot for any gas. The heat capacity term in the energy equation becomes

$$
\rho(\partial h / \partial t)=F^{\prime}(\partial S / \partial t)
$$

where $F^{\prime}$ is the local derivative with respect to $S$ of the auxiliary function. Since $F^{\prime}=$ $\rho C_{\mathrm{p}} / \bar{\kappa}$, the coefficient of the time derivative term is nothing more than the inverse of the instantaneous, temperature-dependent thermal diffusivity of the arc gas. Figures 2 and 3 
indicate that for common gases like nitrogen and argon one can approximate the $(F, S)$ behaviour over a limited range by a straight line. This implies that $F^{\prime}$ is a constant, which we will call $\lambda^{-1}$.

The energy equation can now be rewritten in the following form:

$$
\begin{aligned}
& E^{2} B\left(S-S_{1}\right)+\frac{1}{r} \frac{\partial}{\partial r}\left(r \frac{\partial S}{\partial r}\right)=\frac{1}{\lambda} \frac{\partial S}{\partial t}, \quad 0 \leqslant r \leqslant r_{0}, \quad t>0 \\
& \frac{1}{r} \frac{\partial}{\partial r}\left(r \frac{\partial S}{\partial r}\right)=\frac{1}{\lambda} \frac{\partial S}{\partial t}, \quad r_{0} \leqslant r \leqslant R, \quad t>0 .
\end{aligned}
$$

Here $r_{0}$ is the radial location at which $S=S_{1}$ (see figure 4) and is a function of time. Because of the discontinuous coefficient introduced by the cut-off model for the electrical conductivity, there are two partial differential equations to solve for the heat fiux potential. The solutions of both equations must agree in slope and magnitude at the moving boundary $r_{0}(t)$ and there must be no heat sources within the region of interest. Hence, the three auxiliary conditions are

$$
S_{r}(0, t)=0, \quad S\left(r_{0}{ }^{-}, t\right)=S\left(r_{0}^{-}, t\right)=S_{1}, \quad S_{r}\left(r_{0}{ }^{+}, t\right)=S_{\tau}\left(r_{0}^{-}, t\right)
$$

and, at the tube wall,

$$
S(R, t)=S_{2}
$$

The plus and minus signs denote evaluation of the functions and their derivatives approaching the moving boundary from the outside and inside, respectively. It is the condition regarding continuity of slope that determines the position of the conducting zone radius, which is unknown, a priori. In fact, if the problem is posed in the normal way by specifying the current behaviour, there are three dependent variables $S(r, t), E(t)$ and $r_{0}(t)$. In order to make the problem determinate an additional equation is required. This is provided by Ohm's law,

$$
2 \pi B E(t) \int_{0}^{r_{0}} r\left(S-S_{1}\right) d r=I(t) .
$$

The boundary value problem thus posed, while linear in the variable $S(r, t)$, presents a non-linear system by virtue of the moving boundary and the fact that the electric field strength occurs quadratically in the energy equation. In spite of the simplifications that have been applied to the problem, it is still mathematically formidable. Even if the field strength $E(t)$ and the boundary position $r_{0}(t)$ are prescribed (thereby eliminating the need for some of the above equations), one still is confronted with a partial differential equation with variable coefficients.

In order to arrive at a set of universal equations which apply to any gas we introduce several dimensionless variables, as well as a transformation that eliminates the moving boundary. For the inner region this transformation takes the form

$$
x=r / r_{0}(t), \quad 0 \leqslant r \leqslant r_{0}(t)
$$

and for the outer, annular zone the moving boundary is eliminated by the introduction of

$$
y=(R-r) /\left(R-r_{0}(t)\right), \quad r_{0}(t) \leqslant r \leqslant R .
$$

The range of variation for both of these new independent variables is between zero and one. The dependent variables are made dimensionless by introducing

and

$$
U=\left(S-S_{1}\right) /\left(S_{1}-S_{2}\right), \quad 0 \leqslant r \leqslant r_{0}(t)
$$

Finally, we define

$$
V=\left(S_{1}-S\right) /\left(S_{1}-S_{2}\right), \quad r_{0}(t) \leqslant r \leqslant R
$$

$$
\rho(t)=r_{0}(t) / R, \quad E=B^{1 / 2} R E^{*}, \quad I=I^{*} /\left(S_{1}-S_{2}\right) R B^{1 / 2}, \quad \theta=R^{2} / \lambda, \quad \tau=t / \theta
$$

where $\theta$ is the usual time-scale factor for the heat conduction problem. Earlier, $\rho$ was 
used to denote the gas density, but hereafter it will only refer to the dimensionless moving boundary and should cause no confusion. The starred quantities are the dimensional values and will not appear again. When the above variables are introduced into the boundary value problem a set of universal differential equations is obtained with the particular operating conditions of the arc contained solely within the current forcing function. The problem becomes:

$$
\frac{1}{x} \frac{\partial}{\partial x}\left(x \frac{\partial U}{\partial x}\right)+\frac{1}{2} \frac{d \rho^{2}}{d \tau}\left(x \frac{\partial U}{\partial x}\right)+\rho^{2} E^{2} U=\rho^{2} \frac{\partial U}{\partial \tau}, \quad 0 \leqslant x \leqslant 1, \quad \tau>0
$$

$U_{x}(0, \tau)=0, U(1, \tau)=0$, plus initial condition,

$\left(\frac{\partial^{2} V}{\partial y^{9}}-\frac{1}{\left((1-\rho)^{-1}-y\right\}} \frac{\partial V}{\partial y}\right)-\frac{1}{2}\left(\frac{1-\rho}{\rho}\right) \frac{d \rho^{2}}{d \tau}\left(y \frac{\partial V}{\partial y}\right)=(1-\rho)^{2} \frac{\partial V}{\partial \tau}, \quad 0 \leqslant y \leqslant 1, \tau>0$

$Y(0, \tau)=1, V(1, \tau)=0$, plus initial condition,

and

$$
U_{x}(1, \tau)=-\left(\frac{\rho}{1-\rho}\right) V_{y}(1, \tau)
$$

$$
2 \pi\{\rho(\tau)\}^{2} E(\tau) \int_{0}^{1} x U(x, \tau) d x=I(\tau) .
$$

The non-linearity of the system that is due to the moving boundary can now clearly be seen. In this new fixed domain each differential equation has acquired a term proportional to $d \rho^{2} / d \tau$. Since $\rho$ is one of the dependent variables, the system is non-linear even if the time dependence of the electric field is explicitly known.

It is not strictly necessary, in fact, to consider $E(t)$ as an unknown, but physically it makes more sense to specify the arc current and determine the resulting field strength. For any specified current an arc will be thermally stable but only for certain variations of $E(t)$ will this be true. It would be possible, in fact, to assume an explicit temporal variation of the conducting zone radius and find the temperature profiles that would produce this variation. Then, however, not all of the original equations could be satisfied and one would have to be content with some non-constant tube wall temperature and/or variable tube diameter.

Even the simplest temporal vatiation of $\rho$ that one can devise proves to be too difficult to permit the solution of the boundary value problem. If $d \rho^{2} / d \tau$ is a linear function of $\tau$. for instance, the partial differential equations have variable coefficients and, because of the second term in the left-hand side of each equation, are not separable. Only when $d \rho^{2} d$ t is either zero or a constant of either sign can a closed form solution to the above formulated boundary value problem be readily found. These cases correspond approximately to a high-frequency a.c. arc and a step-modulated d.c. arc, respectively. The latter problem is treated in detail elsewhere (Phillips 1964 Dissertation). The high-frequency a.c. arc is discussed in the following section.

\section{The constant radius a.c. arc}

In the preceding section it was mentioned that the boundary motion specified by $d \rho^{2} / d \tau=0$ is appropriate to a high-frequency a.c. arc. An appeal to physical intuition will indicate why this is so. We note that equation (1) describes a sophisticated heat conduction problem and, as in any time-unsteady conduction problem, the thermal diffusivity is a measure of the speed at which temperature changes can be effected when either a boundary condition or an internal heat generation function is altered. Then, since the conducting zone radius is the locus in the space-time plane of a fixed temperature, its position and rate of change of position is governed by the thermal diffusivity. Since the boundary position is thermally determined, it is reasonable to expect that under some conditions the motion of this interface will be small and its effects unimportant. Specifically, 
if the external conditions that are imposed upon the arc (the current function) are changed so rapidly that the arc cannot readily adjust to these changes, or if the arc is thermally 'slow', there will be little overall variation with time in its structure. A measure of these relative rates of change is the product of the circular frequency $\omega$ of the current waveform and the pseudo-time constant of the arc-tube system, $\theta=R^{2} / \lambda$. When this product is large, implying that either $\omega$ or $\theta$ is large, one expects that there is almost no change in the arc structure.

The intuitive argument given above has been verified theoretically. Analyses by von Engel and Steenbeck (1932), Weizel and Rompe (1949), Frie (1961) and Uhlenbusch (1962 Dissertation) of the linearly modulated d.c. arc all indicate that as the applied frequency of the disturbance is increased, the magnitude of the deviations from the d.c. state becomes vanishingly small. More important, Frie (1961) indicates that the perturbation to the conducting boundary position decreases as $(\omega \theta)^{-3 / 2}$ while centre-line temperature perturbations die out only as $(\omega \theta)^{-1}$, showing the secondary effect of boundary motion at large $\omega \theta$. One expects to observe the same behaviour for the a.c. arc.

We conjecture that at a suitably large value of $\omega \theta$ one can neglect the influence of the moving boundary on the determination of arc structure. It is shown in the sequel how this assumption can be substantiated a posteriori and also how a 'large' $\omega \theta$ can be defined.

If it is presumed that there is effectively no boundary motion, then $d \rho^{2} / d \tau=0$, and equation (1) becomes

$$
\frac{1}{x} \frac{\partial}{\partial x}\left(x \frac{\partial U}{\partial x}\right)+\rho^{2} E^{2} U=\rho^{2} \frac{\partial U}{\partial \tau}, \quad 0 \leqslant x \leqslant 1, \quad \tau>0
$$

$U_{x}(0, \tau)=0, U(1, \tau)=0$, plus initial condition

$$
2 \pi \rho^{2} E \int_{0}^{1} x U(x, \tau) d x=I_{\mathrm{m}} \cos (\omega \theta \tau)
$$

where the harmonic current waveform has been specified. Since the boundary motion is known, there is no need for either the annular differential equation or the remaining boundary and compatibility conditions.

Assuming a solution for equation (2) of the form

yields the spatial solution

$$
U(x, \tau)=X(x) T(\tau)
$$

$$
X(x)=C \mathrm{~J}_{0}(\beta x) \text {. }
$$

One finds that for non-trivial solutions the separation constant $\beta$ must assume the values of the zeros of the Bessel function. After some manipulation we find the solution of the temporal equation to be

$$
T^{2} \exp \left(\frac{2 \beta^{2} \tau}{\rho^{2}}\right)=\frac{\beta^{2} I_{\mathrm{m}}^{2}}{2 \pi^{2} \rho^{4} \mathrm{~J}_{1}^{2}(\beta)} \int_{0}^{\tau} \exp \left(\frac{2 \beta^{2} \alpha}{\rho^{2}}\right) \cos ^{2}(\omega \theta \alpha) d a+T_{\mathrm{i}}^{2} .
$$

For the initial conditions it is convenient to use the properties of a d.c. arc that is carrying a current $I_{\mathrm{m}}$, equal to the peak value of the a.c. current level. In the d.c. arc all timeunsteady terms vanish, and it is not difficult to obtain

$$
U_{\mathrm{i}}(x)=U_{\mathrm{m}} \mathrm{J}_{0}\left(\beta_{1} x\right)
$$

Using the compatibility condition allows one to obtain the solution to the annular equation in the form

$$
V_{\mathrm{i}}(y)=U_{\mathrm{m}} \beta_{1} \mathrm{~J}_{1}\left(\beta_{1}\right) \ln \left(\frac{1-(1-\rho) y}{\rho}\right) .
$$

Since the initial distribution of heat flux potential is identical to the first eigenfunction of the present boundary value problem, the first Fourier coefficient is unity and all others are 
identically zero. Evaluating the integral in equation (3), applying the initial conditions, and letting $\tau \rightarrow \infty$ leads one to the result

$$
U(x, \tau)=\frac{I_{\mathrm{m}} \mathrm{J}_{0}\left(\beta_{1} x\right)}{2 \sqrt{2 \pi \rho \mathrm{J}_{1}\left(\beta_{1}\right)}}\{1+\cos \gamma \cos (2 \omega \theta \tau-\gamma)\}^{1 / 2}
$$

where $\tan \gamma=(\omega \theta) \rho^{2} / \beta_{1}^{2}$.

It was mentioned earlier that the above solution would be expected to apply to the situation where the conducting boundary motion is slight and consists of a small oscillation about some mean value. One would not expect that mean value to be the d.c. value so the solution is not complete until a proper a.c. radius has been defined. For large $\omega \theta, \gamma \rightarrow \pi / 2$, $\cos \gamma \rightarrow 0$, and after referring all quantities to their d.c. counterparts, equation (4) becomes

$$
U(x)=\frac{\sqrt{ } 2}{2} \frac{U_{\mathrm{m}}}{\left(\rho / \rho_{\mathrm{dc}}\right)} J_{0}\left(\beta_{1} x\right) .
$$

One sees that were it not for the difference in conducting radii the heat flux distribution of the high-frequency limit a.c. arc would be the root-mean-squared value of the corresponding d.c. column. As it is, by analogy with the d.c. solution, one finds the radii to be related by

$$
\frac{\rho}{\ln \rho}=\frac{\sqrt{2}}{2} \frac{\rho_{\mathrm{dc}}}{\ln \rho_{\mathrm{dc}}}
$$

From equation (4) it is possible to obtain the electric field waveforms that correspond to this high-frequency arc. With the help of Ohm's law one finds

$$
E=\sqrt{ } 2 \frac{\beta_{1}}{\rho} \frac{\cos (\omega \theta \tau)}{\{1+\cos \gamma \cos (2 \omega \theta \tau-\gamma)\}^{1 / 2}}
$$

which applies only for the assumed cosine current waveform. Notice that as $\omega \theta$ becomes very large, implying that $\cos \gamma \rightarrow 0$, the voltage waveform precisely follows the current input and is directly proportional to it, just as in an ordinary resistor. In figure 5, by plotting equation (5) for values of $y$ from zero to $\pi / 2$ we show all possible shapes that can be assumed by the electric field waveform.
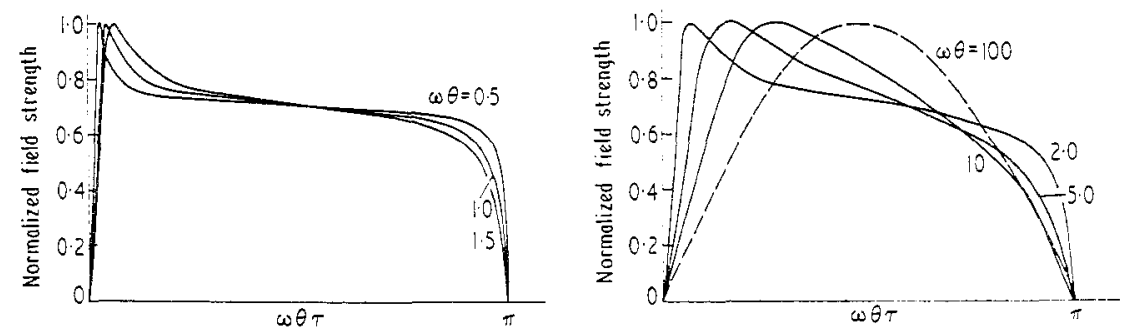

Figure 5. Electric field waveforms for a constant radius a.c. arc.

Any analysis of the a.c. arc column must be capable of predicting two well-known features that are characteristic of small values of $\omega \theta$. Shortly after current zero passage a large voltage spike appears which is known as the re-ignition peak. The voltage then drops sharply to a lower value, remains nearly constant for much of the half-cycle, and then rises slightly to a second peak before falling to zero at the next current zero passage. This second voltage spike is called the extinction peak. In practice, the value of $\omega \theta$ at which these features occur is certainly less than 5 and probably close to unity.

Figure 5 indicates that none of the theoretical, low $\omega \theta$ waveforms bears the proper resemblance to reality. The re-ignition peaks are too low relative to the plateau values to be realistic and an extinction peak never develops. It would, of course, be quite surprising if this analysis agreed with experiment because for a thermally-fast arc the effect of the 
moving boundary must surely be important. In addition, at low frequencies a dynamic arc can almost follow its corresponding d.c. characteristic and for the present model the d.c. electric field is independent of the current. Hence an extinction peak could never develop for the constant radius a.c. arc and the re-ignition peak would never have the proper shape. Then, as was originally stipulated, the solution can only apply to a thermally-slow or highfrequency arc.

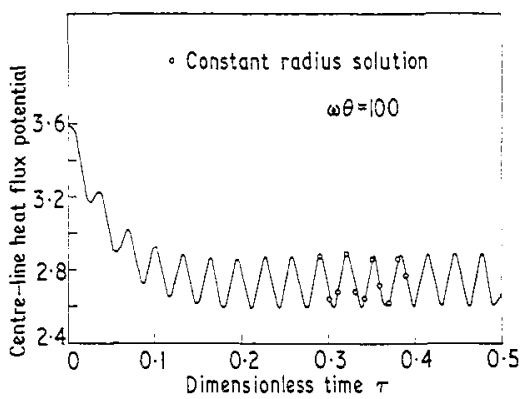

Figure 6. Comparison of centre-line heat flux potential behaviour obtained from the constant radius model and from numerical computation, $\omega \theta=100$.

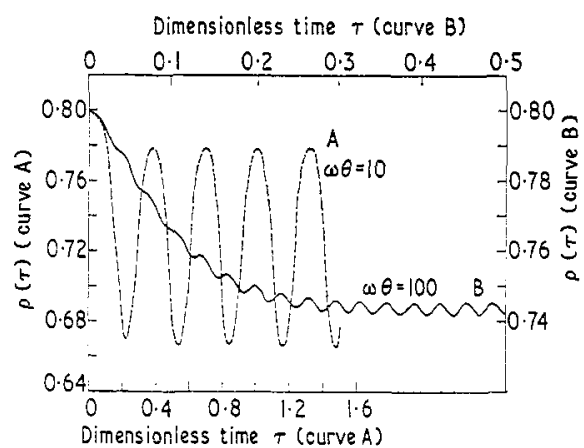

Figure 7. Exact behaviour of the conducting zone radius for $\omega \theta=10$ and $\omega \theta=100$.

In figure 6 we compare the variation of centre-line heat flux potential for the 'exact' arc and the 'approximate' arc (equation (4)) for $\omega \theta=100$ and $\rho_{\mathrm{dc}}=0.80$. The exact solution to equation (1) was obtained numerically by a method described in the next section. The agreement is excellent, indicating that 100 is a 'large' value of the frequency parameter. A similar comparison for the same $\rho_{\mathrm{dc}}$ and $\omega \theta=10$ indicates the agreement is satisfactory but not as good as in the first case. Figure 7 shows why there begins to be a discrepancy between the two solutions. Plotted there is the variation in $\rho(\tau)$, starting from a d.c. state and relaxing to a quasi-steady a.c. value. For $\omega \theta=100$, the radius undergoes only a $\frac{1}{2} \%$ oscillation about its mean value while the central heat flux potential has about a $5 \frac{1}{2} \%$ variation. For $\omega \theta=10$ the oscillations are $7 \%$ and $40 \%$, respectively, so that the moving boundary has more than a second order effect in that case.

A slightly less general solution to the constant radius a.c. arc problem has been obtained by Edels and Fenlon (1965) yielding essentially the same results as the present analysis. They have also studied the 'filled-tube' arc, as they call it, for current waveforms other than the harmonic one used here.

In the next section it is shown how a numerical solution to equation (1) can be obtained so that the low values of $\omega \theta$ can be examined.

\section{The numerical solution of the boundary value problem}

\subsection{The finite difference method}

Except for two or three special cases, it has not been possible to find an analytical solution to the boundary value problem given by equation (1). It was decided, therefore, to attempt to solve the system of equations numerically for an a.c. positive column which is sustained by a sine wave of current. The numerical technique adopted here is closely related to that used to obtain solutions to partial differential equations on analogue computers. There, one first applies a finite difference scheme to the space-like variables, leaving a series of ordinary differential equations to be solved in the usual way on an analogue computer (MacKay and Fisher 1962). Fox (1962) also mentions this procedure and suggests that one might try solving the resulting ordinary differential equations on a digital rather than 
analogue computer by some simple forward integration technique such as the Runge-Kutta method. The accuracy is no better than that obtainable with an analogue solution, but it is usually simpler to implement the digital process.

Finite difîerence schemes are discussed in many books on numerical analysis. By using the first order central difference method a rather high degree of accuracy can be obtained without the expense of undue mathematical sophistication. This method has been applied to equation (1), yielding the results discussed in the next section.

\subsection{Numerical results and discussion}

From the d.c. solution one finds that for $\rho_{\mathrm{dc}}=0.8, I_{\mathrm{m}}=9 \cdot 4$. Depending upon the particular type of gas and constrictor size for which one desires physical results, this could represent current levels from a few amps to perhaps 100 amps. With this parameter fixed, $\omega \theta$ has been varied between 1 and 100 . Three sets of data corresponding to high, intermediate and low frequencies are examined.

The most interesting aspect of the high-frequency column, $\omega \theta=100$, is the behaviour of the concucting boundary. This has already been presented in figure 7 . It is not too instructive to show the other results for this case because they agree with those found from the constant radius analysis of the previous section. In general, the arc behaves as though it were an ordinary resistor.

At lower effective frecuencies the entire character of the arc changes. Since the thermal properties can follow the externally applied variations more faithfully, there are large changes in the heat fiux potential, conducting zone radius, and other variables. Due to the large non-linear changes in thermal conditions that occur, there no longer exists the proportionality between the applied current and the arc voltage that one finds at higher $\omega \theta$.

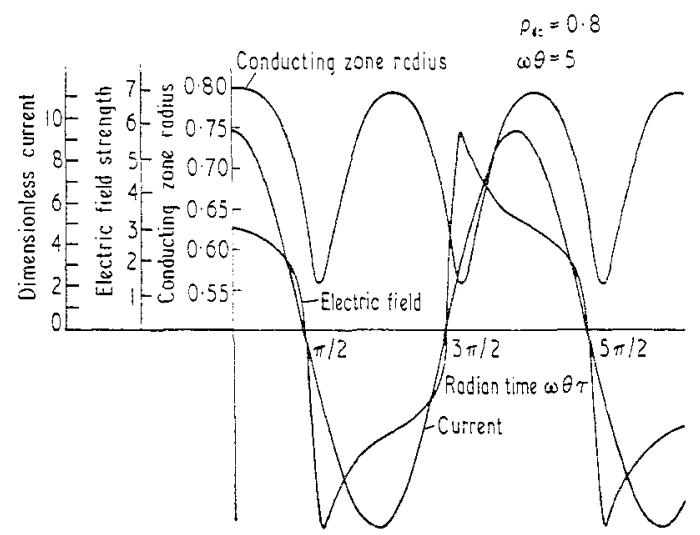

Figure 8. Theoretical waveforms of conducting zone radius, electric field strength and dimensionless current for $\omega \theta=5$.

The electric field and conducting radius waveforms for $\omega \theta=5$ are shown in figure 8 The electric field waveform displays a sharply rising behaviour immediately following the current zero passage. Physically, this obtains from the arc having lost considerable energy (and having gained resistance) during the interval when the current was low. The small time constant indicates that the arc stores energy poorly and rapidly loses the qualities of a good conductor of electricity. Since the current is specified, the electric field required to produce this electron flux becomes large and remains so until enough energy has been added to appreciably increase the column conductance. The strong departure of the $E(\tau)$ waveform from a sine curve indicates the presence of harmonics higher than the fundamental. This leads to some interesting speculations on the power factor of an a.c. arc, a subject to be discussed later. 
Finally, consider the characteristics of a low-frequency arc, as depicted in figure 9 . Here $\omega \theta=1 \cdot 0$, a value close to that typically obtained in the laboratory. One notices immediately the broad maximum for $\rho(\tau)$. The small value of $\omega \theta$ used for these calculations implies that the d.c. characteristic (the $(E, I)$ curve) can nearly be followed. From the d.c. solution found in the previous section one finds that during the high eurrent portion of the sine wave input conditions are such that $d \rho / d I$ is very small. Hence the flat top for the $\rho(\tau)$ curve. Near current zero, however, the static curve can no longer be followed and the sharply rising and falling portions of the radius waveform are dictated by dynamic considerations.

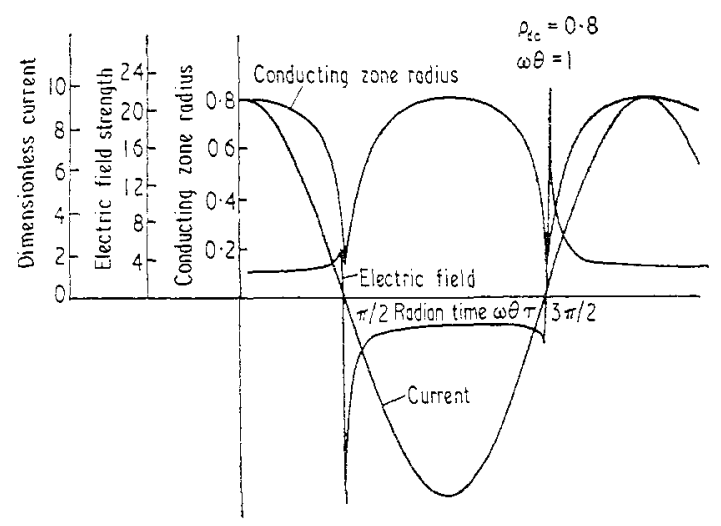

Figure 9. Theoretical waveforms of conducting zone radius, electric field strength and dimensionless current for $\omega \theta=1$.

The electric field waveform exhibits an immense re-ignition transient immediately following the zero current passage. The arc suffers a rapid loss in conductance when it is momentarily extinguished so that a large field is required to drive the specified current. As the column gains energy again from the Joule heating, the field strength drops to a value that is comparable to the d.c. level and remains nearly constant for over $50 \%$ of the halfcycle. This plateau behaviour is explained by reference to the static characteristic shown in figure 10 where we see that at high current levels the arc voltage varies only slightly with the current. A low-frequency a.c. arc can closely follow the static characteristic until the time comes for current reversal. At its lowest point the a.c. arc burns with a voltage somewhat

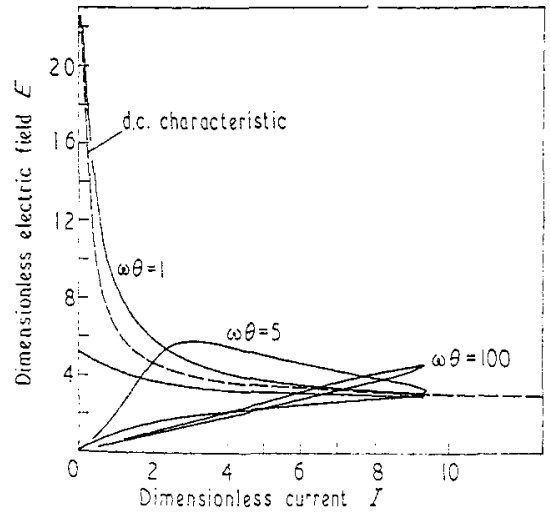

Figure 10. Theoretical dynamic and static arc characteristics.

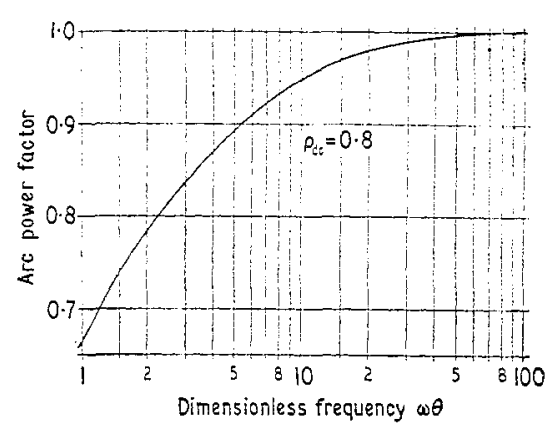

Figure 11. Theoretical power factor plotted against $\omega 0$ for an isolated a.c. arc. 
below the corresponding d.c. level. This is a dynamic effect due to the a.c. arc actually overheating as a result of the initial voltage transient.

The behaviour of a.c. and d.c. arcs can be clearly shown in a cyclogram or dynamic $(E, I)$ characteristic. Such a plot appears in figure 10, with only the positive half-cycle of the a.c. arcs being shown. At high frequencies the near-ohmic, positive characteristic is in evidence, while for $\omega \theta=1$ the falling d.c. characteristic is closely approximated. In fact, this close adherence to the d.c. curve brings about a voltage rise before current zero passage that has been observed experimentally, the extinction peak. For the conditions assumed in the present numerical calculations, it appears that $\omega \theta$ must be less than 1.5 before an extinction peak develops. With an external circuit or different current magnitudes this conclusion may be altered considerably.

Compared with the waveform for the high-frequency arc, one notes from figure 9 that the character of the electric field waveform has changed from that of a pure sine curve to that of nearly a square wave. The arc, being a non-linear element, introduces harmonics into a circuit that are not originally there; the current is, after all, a pure waveform. It is interesting to examine one of the consequences of the presence of harmonics in a circuit element that are higher than fundamental.

If one retains the usual definition of power factor, one finds that the power factor for an a.c. arc carrying a sine wave of current can be significantly different from unity. This statement applies for the arc alone, independent of any circuit. For the usual a.c. circuit with linear elements it is easy to understand a non-unity power factor; some amount of energy is always stored in a capacitor or inductor. Here, however, all input energy is dissipated in the arc and to understand the existence of a power factor less than one it is necessary to re-examine the concept of effective circuit variables.

It is well known that special considerations must be given to the analysis of a circuit that contains non-fundamental harmonics. If the average power of a circuit element is defined by

$$
W=\frac{1}{T} \int_{0}^{T} E I d \tau
$$

where $T$ is the fundamental period, one can show that a value of $W$ different from zero is obtained only by the combination of a voltage and current component of the same frequency. In the present case $I(\tau)$ is a pure sine wave so the amplitude of its first and only harmonic is simply $I_{\mathrm{m}}$. The voltage waveform, however, is rich in high harmonics, none of which will contribute to the average power. Upon integrating the above expression one obtains

$$
W=\frac{1}{2} E_{\mathrm{m}_{1}} I_{\mathrm{m}}
$$

where $E_{\mathrm{m}_{1}}$ is the magnitude of the first voltage harmonic. On the other hand, the usual definition of effective voltage requires that

$$
E_{\mathrm{efi}}=\frac{1}{2} \sqrt{ } 2\left(E_{\mathrm{m}_{1}^{2}}{ }^{2}+E_{\mathrm{m}_{2}}{ }^{2}+\ldots\right)^{1 / 2} .
$$

As usual, $I_{\mathrm{eff}}=(\sqrt{ } 2 / 2) I_{\mathrm{m}}$ and it is easy to see that if the voltage wave has a non-fundamenta harmonic of even the most minute magnitude, the power factor of the arc, $W / I_{\text {eff }} E_{\text {eff }}$ cannot be precisely one. Physically, the large arc voltage that is required to overcome the high resistance that follows extinction is ineffectively used to produce power. The current is very low at this point and the power added to the arc is trivial compared to that potentially obtainable from a situation where the voltage and the current have the same harmonic content. In figure 11 the variation in arc power factor with the effective frequency $\omega \theta$ is shown. When one is trying to dissipate power by means of an a.c. arc it is clear that the higher the value of $\omega \theta$, the more efficient is the utilization of the available power supply. This non-linear effect is penalizing to the arc heater designer who uses a.c. power.

To complete this section we show a few heat flux potential and temperature profiles that result from the numerical computations. For the high-frequency arc there is virtually no deviation from some mean value profile, which in turn closely resembles the d.c. structure. 
The latter is shown in figure 12 for an initial dimensionless arc radius of 0.8 . Also shown is an intermediate profile for the $\omega \theta=1$ case, as well as the heat flux potential distribution at the lowest point in the cycle. Bessel function profiles, appropriate to d.c. only, are included for comparison. It is seen that the dynamic effects of the a.c. arc do not cause a significant deviation from these curves.

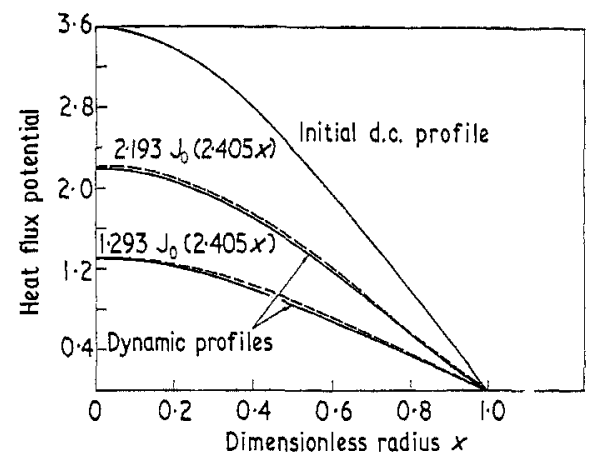

Figure 12. Theoretical heat flux potential profiles for the electrically conducting region of an a.c. arc.

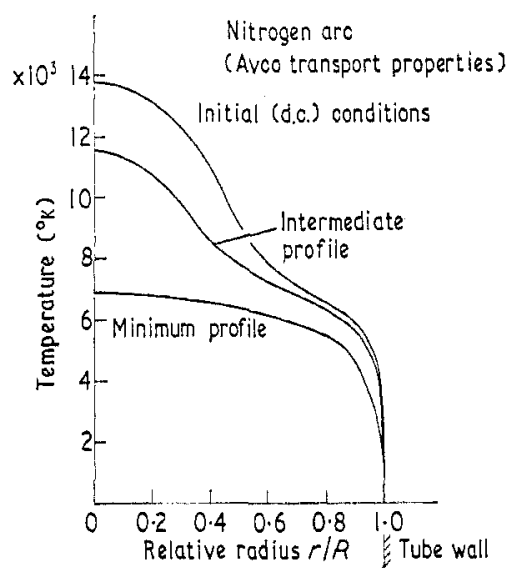

Figure 13. Theoretical temperature profiles for a nitrogen a.c. arc.

In figure 13 temperature profiles for nitrogen, corresponding to the heat flux potential curves of figure 12, are shown. One can see the transition from the low current "coreless' arc to the temperature distribution that denotes core formation in diatomic gases. This well-known feature is a consequence of the dissociation peak in the thermal conductivity curve for molecular gases.

The measurements of the characteristics of a.c. arcs that are reported in the literature have all been performed on free burning arcs, where the effects of natural convection are not accurately known. A comparison of the present theory with these results would not be meaningful. There are, however, measurements of the wall-stabilized a.c. column made by the author (Phillips 1964 Dissertation) that can be directly compared with the theoretical predictions. The results of this experimental programme will be described in detail in a later paper. Here we note that for conditions corresponding to the assumptions made at the outset there is excellent agreement between the experimental and theoretical electric field waveforms. Not only is the magnitude of the column voltage in good agreement with theory but the qualitative change in wave shape with varying $\omega \theta$ is accurately predicted. The conclusions one can draw from this agreement are discussed in the next section.

\section{Summary}

The equations that describe the behaviour of a time-unsteady plasma column have been simplified by neglecting energy transfer by radiation and convection. Without the inclusion of radiation only low current phenomena can be studied. Furthermore, the thermal diffusivity of the plasma was assumed to be constant and a simplified expression for the electrical conductivity was adopted. Thus, the energy equation was cast into a universal form, requiring the specification of only two parameters to obtain solutions. The governing set of equations was solved for a suitable range of these parameters for an a.c. arc that is sustained by a sinusoidal current.

In view of the good agreement between the theoretically derived voltage waveforms and those found from a specially designed a.c. cascade, it is concluded that the behaviour of the low current a.c. arc can be described by the solution of a non-linear heat conduction problem. 
Moreover, the solutions of this problem, while complicated, have the advantage over older theories (Cassie 1939, Mayr 1943) that they can be directly related to measurable or calculable quantities that characterize a particular gas. This correspondence occurs through the two parameters $\omega \theta$ and $I_{\mathrm{rn}}$, which determine the arc dynamic response and current level, respectively.

In order to study the properties of dynamic arcs at higher current levels, several limitations of the present analysis must first be removed. The radiative transfer term should be included in the energy equation if either higher currents or elevated pressures are to be studied. Also, at some current level the strength of the self-magnetic field will become significant and a coupling of the energy equation with Maxwell's equations will result.

The single straight line approximation to the electrical conductivity function is not suitable for high current levels. Not only will there be a quantitative error in the computed arc properties but a fundamental feature of high current tube arcs will not be predicted--the minimum point and the rising part of the $(E, I)$ curve.

By approximating the $(F, S)$ curve by a single straight line we have assumed a constant thermal diffusivity for the arc gas. Actually, since such extremes of terrparature exist in the arc, there is always a variation of $\lambda$ throughout the tube cross section. This variation need not produce significant effects (as is apparently the case with argon) but it is known to be important for arcs burning in diatomic gases, where a dissociation core is formed (Christmann and Hertz 1966, Edels and Kimblin 1965).

Finally, it is not clear that the effects of radial convection in the time-unsteady arc can always be ignored. Studies are now under way to examine these effects as well as those associated with higher current levels, mentioned above.

\section{References}

Bishop, D. O., 1954, Proc. Instn Elect. Engrs, 101, 18-26.

Cassie, A. M., 1939, Conf. Int. des Grands Réseaux Électriques à Haute Tension, Paris (Paris: CIGRE), Paper 102, pp. 1-14.

Christman, H., and HerTz, W., 1966, Proc. 7th Int. Conf. on Phenomena in Ionized Gases, Belgrade, 1965 (Belgrade: Gradevinska Knjiga).

Drellishak, K. S., Aeschlimai, D. P. and Cambel, A. B., 1964, Arnold Eng. Dev. Cir. Rep. AEDC-TDR-64-12.

Edels, H., and EtTnger, Y., 1962, Proc. Insth Elect. Engrs, 109A, 89-98.

Edels, H., and FeNLox, F. H., 1965, Brit. J. Appl. Phys., 16, 219-30.

Edels, H., and Kimblin, C. W., 1965, Plasma Symp. XXth Int. Congress on Pure and Applied Chemistry, Moscow:

VON ExGel, A., and STEexBeck, M., 1932, Elehtrische Gasentladungen, ihre Physik und Technik (Berlin: Springer).

Fnkel Nburg, W., and Maecker, H., 1956, Handb. d. Phys., 22, 254 (Berlin: Springer-Verlag).

Fox, L., 1962, Numerical Solution of Ordinary and Partial Differential Equations (Reading, Mass.: Addison-Wesley).

FRIE, W., 1961, $Z$. angew. Phys., 13, 99-102.

Frind, G., 1960, $Z$. anger. Phys., 12, 231-7.

Hirscheelder, J. O., CURtiss, C. F., and BiRd, R. B., 1954, Molecular Theory of Gases and Liquids (New York: John Wiley).

JoHn, R. R., and B.ADE, W. L., 1961, Amer. Rocket Soc. J., 31, 4-17.

JoHs, R. R., et al, 1963. Aeronautical Sistems Div. Rep. ASD-TDR-62-729.

LOH, O., 1959, Arch. Elektrotech., 44, 203.

MacKay, D. M., and Fischer, M. E., 1962, Analogue Computing at Ultra-High Speed (London: Chapman and Hall).

MAECKer, H., 1959, Z. Phys., 157, 1-29.

MAYR, O., 1943, Arch. Elektrotech., 37, 588-608.

NoEske, H., 1957, Arch. Elektrotech., 43, 114-33.

Phillips, R. L., 1964a, Aerospace Res. Lab. Rep. ARL 64-9.

1964b, AGAR Dograph, 84, 797-843.

Schmitz, G., 1950., Z. Naturf., 5a, 571.

WEIZEL, W., and ROMPE, R., 1949, Theorie elektrischer Lichtbögen und Funken (Leipzig: Barth). 